\title{
A novel genetic algorithm for solving the clustered shortest-path tree problem
}

\section{Ovidiu Cosma, Petric ă C. Pop and IOAna Zelina}

\begin{abstract}
.
The clustered shortest-path tree problem is an extension of the classical single-source shortest-path problem, in which, given a graph with the set of nodes divided into a predefined, mutually exclusive and exhaustive set of clusters, we want to determine a shortest-path spanning tree from a given source to all the other nodes of the graph, with the property that each cluster should induce a connected subtree. The investigated problem proved to be NP-hard and therefore we proposed an efficient genetic algorithm in order to solve it. The preliminary computational results reported on a set of benchmark instances from the literature proved that our proposed solution approach yields high-quality solutions within reasonable running times.
\end{abstract}

\section{REFERENCES}

[1] Binh, H. T. T., Thanh, P. D., Trung, T.B. and Thao, L. P., Effective multifactorial evolutionary algorithm for solving the cluster shortest path tree problem, in Proc. of IEEE Congress on Evolutionary Computation (CEC), pp. 819-826, 2018

[2] Binh, H. T. T., Thanh, P. D. and Thang, T. B., New approach to solving the clustered shortest-path tree problem based on reducing the search space of evolutionary algorithm, Knowledge-Based Systems, 180 (2019), 12-25

[3] Demange, M., Monnot, J., Pop, P. C. and Ries, B., On the complexity of the selective graph coloring problem in some special classes of graphs, Theor. Comput. Sci., 540-541 (2014), 82-102

[4] D'Emidio, M., Forlizzi, L., Frigioni, D., Leucci, S. and Proietti, G., On the clustered shortest-path tree problem, in Proc. of Italian Conference on Theoretical Computer Science, pp. 263-268, 2016

[5] D’Emidio, M., Forlizzi, L., Frigioni, D., Leucci, S. and Proietti, G., Hardness, approximability and fixedparameter tractability of the clustered shortest-path tree problem, J. Comb. Optim., 38 (2019), 165-184

[6] Fidanova, S. and Pop, P.C., An improved hybrid ant-local search for the partition graph coloring problem, J. Comput. Appl. Math., 293 (2016), 55-61

[7] Fischetti, M., Salazar-Gonzales, J. J. and Toth, P., A Branch-and-Cut Algorithm for the Symmetric Generalized Traveling Salesman Problem, Oper. Res., 45 (1997), No. 3, 378-394

[8] Ghiani, G. and Improta, G., An efficient transformation of the generalized vehicle routing problem, Eur. J. Oper. Res., 122 (2000), 11-17

[9] Thanh P. D., CluSPT instances. Mendeley Data v3. https://doi.org/10.17632/b4gcgybvt6.3 (2019)

[10] Myung, Y. S., Lee, C. H. and Tcha, D. W., On the generalized minimum spanning tree problem, Networks, 26 (1995), 231-241

[11] Pintea, C., Chira, C., Dumitrescu, D. and Pop, P. C., Sensitive ants in solving the generalized vehicle routing problem, Int. J. Comput. Commun., 6 (2011), No. 4, 734-741

[12] Pop, P. C., Generalized Network Design Problems, Modelling and Optimization De Gruyter, Germany, 2012

[13] Pop, P. C., Matei, O. and Sabo, C., A hybrid diploid genetic based algorithm for solving the generalized traveling salesman problem, in Proc. of HAIS 2017, Lect. Notes Comput. Sci., 10334 (2017), 149-160

[14] Pop, P. C., Matei, O., Sabo, C. and Petrovan, A., A two-level solution approach for solving the generalized minimum spanning tree problem, Eur. J. Oper. Res., 265 (2018), No. 2, 478-487

Received: 27.03.2020; In revised form: 01.07.2020; Accepted: 07.07.2020

2010 Mathematics Subject Classification. 05C85, 68T20.

Key words and phrases. single-source shortest-path problem, clustered shortest-path tree problem, genetic algorithms.

Corresponding author: Petrică C. Pop; petrica.pop@cunbm.utcluj.ro 
[15] Pop, P. C., Fuksz, L., Horvat Marc, A., and Sabo, C., A novel two-level optimization approach for clustered vehicle routing problem, Computers \& Industrial Engineering, 115 (2018), 304-318

[16] Pop, P. C., The generalized minimum spanning tree problem: an overview of formulations, solution procedures and latest advances, Eur. J. Oper. Res., 283 (2020), No. 1, 1-15

[17] Thanh, P. D., Dung, D. A., Tien, T. N. and Binh, H. T. T., An Effective Representation Scheme in Multifactorial Evolutionary Algorithm for Solving Cluster Shortest-Path Tree Problem, in Proc. of IEEE Congress on Evolutionary Computation (CEC), art. No. 8477684, 2018

[18] Thanh, P. D., Binh, H. T. T., Dac, D. D., Binh Long, N. and Hai Phong, L. M., A Heuristic Based on Randomized Greedy Algorithms for the Clustered Shortest-Path Tree Problem, in Proc. of IEEE Congress on Evolutionary Computation (CEC), art. no. 8790070, pp. 2915-2922, 2019

Department of Mathematics and Computer Science

TECHNICAL UNIVERSITY OF CLUJ-NAPOCA

North University CENTRE AT BAIA MARE

VICTORIEI 76, RO-430122 BAIA MARE, ROMANIA

Email address: ovidiu. cosma@cunbm. utcluj.ro

Email address: petrica.pop@cunbm.utcluj.ro

Email address: ioana.zelina@cunbm.utcluj.ro 